Tagungsberichte

* Lehrer.Bildung.Medien
* Medienpädagogische Grundbildung für pädagogische Fachkräfte

\section{LEHRER.BILDUNG.MEDIEN - Herausforderungen für die Entwicklung von Schule}

Tagung am 1. Oktober 2014, Technische Universität Kaiserslautern, Zentrum für Lehrerbildung

GESINE KULCKE

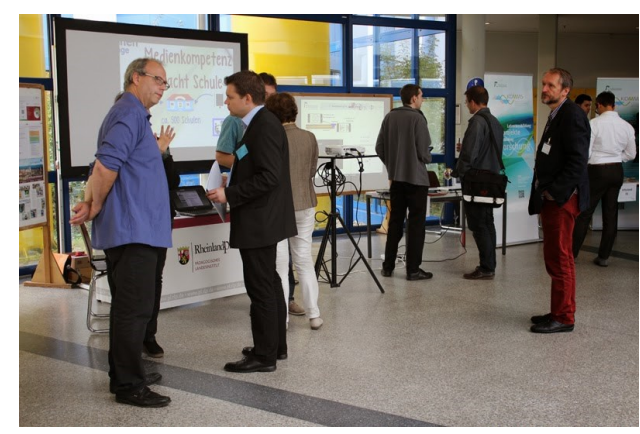

Schon die Ausstellung aktueller Forschungsprojekte im Eingangsbereich des Tagungsgebäudes machte deutlich, dass sich Schule nur dann weiterentwickeln kann, wenn sich auch die Lehrerbildung weiterentwickelt. Dazu beitragen wollen u.a. Thomas Prescher und seine Kollegen von der TU Kaiserslautern. Sie untersuchen, wie Onlineseminare, die für das Lehramt angeboten werden, gestaltet sein müssen, damit sie selbstgesteuertes Lernen fördern. Anja Schwedler beschäftigt sich dagegen mit der Frage, wie medienpädagogische Professionalisierungsprozesse von Lehramtsstudierenden verlaufen, die an einer an der Universität Hamburg angebotenen Forschungswerkstatt zum Thema Neue Medien teilnehmen und hier eigene Forschungsarbeiten durchführen. Im Kontext der Lehrerbildung interessiert zudem nach wie vor die Frage nach den medienbezogenen Einstellungen, die Lehramtsstudierende mitbringen: So untersucht Sue-Ann Bäsler von der Freien Universität Berlin medienbezogene Einstellungen als Bedingungen für die Ausbildung medienpädagogischer Kompetenz bei angehenden Lehrpersonen und fragt, welchen Stellenwert Medien für angehende Lehrerinnen und Lehrer in Bezug auf Vorbereitung und Ausübung des Lehrerberufs, die Gestal- tung von Unterricht und damit den Lernerfolg von Kindern und Jugendlichen haben.

Stefan Aufenanger und Jasmin Bastian von der Universität Mainz stellten ihr Forschungsprojekt in einer der zehn Foren vor, die während der Tagung angeboten wurden: Sie wollen wissen, welche Einstellung und Weltbilder angehende Lehrpersonen mitbringen, und wie diese im Laufe der drei Phasen des Lehramtsstudiums berücksichtigt werden können. Sie gehen davon aus, dass Medienbildung an Schulen nur dann stärker verankert werden kann, wenn es entsprechend ausgebildete Lehrpersonen gibt, die den Einsatz digitaler Medien wichtig und sinnvoll finden.

Für ihre im Sommersemester 2014 begonnene Studie Medienberogene Einstellung und Weltbilder von (angehenden) Lehrpersonen haben sie sowohl Lehrerinnen und Lehrer an Schulen befragt, die an einem TabletProjekt teilnehmen, als auch Studierende, die im ersten und im dritten Semester sowie im Master auf Lehramt für Gymnasium studieren. Erste Ergebnisse der Studie, die bis zum Wintersemester 2016/2017 weitergeführt werden soll, machen deutlich, dass die befragten Lehrerinnen und Lehrer digitalen Medien positivere Effekte unterstellen als die Lehramtsstudierenden, wobei hier berücksichtigt werden muss, dass die befragten Lehrerinnen und Lehrer von einer Schule kommen, an der zurzeit ein TabletProjekt durchgeführt wird, das womöglich ihre Haltung beeinflusst.

Die negativen Effekte, die Medien zugeschrieben werden, gleichen denen, die aus der Presse bekannt sind und unterstellen Medien vor allem eine süchtig machende Wirkung, wobei die Studierenden, vor allem Kinder für suchtgefährdet halten und weniger sich selbst: Für sich selbst sehen sie in den digitalen Medien besonders Arbeitsund Kommunikationsmittel.

Erste Ergebnisse, die Sue-Ann Bäsler von der Freien Universität Berlin aus den Interviews ableitet, die sie mit Studierenden geführt hat, weisen darauf hin, dass sich medienbezogene Einstellungen im Laufe des Studiums entwickeln und sich ein medienbezogenes Angebot hier positiv auswirken kann. Studierende, die Medienseminare be- 
sucht haben, zeigen größeres Interesse an Neuen Medien für ihren Beruf, erklärt Sue-Ann Bäsler. Ein bestimmtes Muster, dem der mediale Habitus von Lehramtsstudierenden folgt, kann sie nicht erkennen. Er wirke aber, so Sue-Ann Bäsler, nicht mehr so traditionell wie in vergangenen Studien zum medialen Habitus beschrieben. Ähnliches lässt sich auch aus der Onlinebefragung der Pädagogischen Hochschule Zürich herauslesen, mit der Walter Scheuble, Sara Signer und Heinz Moser Ende 2012 und Anfang 2013 Studierende der PH danach gefragt haben, wie sie ihre eigenen Medienkompetenzen einschätzen, welche Einstellung sie zu Medien haben, und wie sie selbst Medien nutzen. Das Ergebnis: die Studierenden zeigen eine hohe Offenheit und Pragmatik gegenüber digitalen Medien.

Wie die Studie aus Mainz so soll auch die Studie in Zürich weitergeführt bzw. daran anknüpfend weitergearbeitet werden, wie Klaus Rummler auf der Tagung in Kaiserslautern berichtete: Ziel ist die Ausarbeitung eines Rahmens für individuelle Hochschulentwicklung, der sich auf andere Hochschulen übertragen lässt. Die Entwicklung eines solchen Rahmens soll von den persönlichen Lernumgebungen der Studierenden ausgehen, die Lernaktivitäten aus ihrer Sicht beschreiben und damit Hinweise darauf geben, welche Rolle Medienangebote der Hochschule in ihrer persönlichen Lernumgebung spielen. Um eine Entwicklung nachzeichnen und herausarbeiten zu können, was Berufseinsteiger von der Ausbildung in den Lehrbetrieb mitnehmen, sollen einzelne Studierende über mehrere Jahre begleitet werden.

Auf die Schnittstelle Studium und Berufspraxis gingen auch Hannah Hoffmann und Petra Bauer von der Universität Mainz in dem Forum Medienbildung im Übergang ein: Sie präsentierten von Studierenden entwickelte Materialien für selbstreguliertes und praxisorientiertes Lernen, mit denen die Studierenden bereits während des Studiums an Schulen gehen und arbeiten, um medienpädagogische Inhalte und Kompetenzen, die sie im Studium erworben haben, erproben zu können. Claudia Bremer von der Universität Frankfurt stellte im gleichen Forum das hessische Projekt Lehr@mt vor. Es verankert die Medienkompetenzbildung in allen drei Phasen der Lehrerbildung. Aufgeschlüsselt wird die Medienkompetenz in Entwicklung, Einsatz und Evaluation von digitalen Lerninhalten und Unterrichtsmaterial, sowie Methoden und Unterrichtsszenarien mit Medien. Damit liegt der Fokus auf der Mediendidaktik. Kooperationspartner des Projekts sind entsprechend Fachdidaktiken wie die Didaktik der Chemie, der Geschichte, der katholischen Religion und Mathematik. Im Laufe des Projektes haben Studierende zahlreiche Webquests und andere Lernmaterialien entwickelt und in Schulen eingesetzt. Entstanden ist im Rahmen des Projekts Lehr@mt zudem das Medienkompetenz- zertifikat, das für das Zertifikat akkreditierte Fächer der Universität Frankfurt kooperativ ausbringen.

Auch das Zertifikat bezieht alle drei Phasen der Lehrerbildung ein. Es umfasst drei Kompetenzbereiche: die fachliche Kompetenz, die Anwendungskompetenz und die Handlungs- und Gestaltungskompetenz, die sich auf die Unterrichtsgestaltung mit Neuen Medien bezieht. Die erziehungswissenschaftliche Perspektive wird in einer Ringvorlesung berücksichtigt, in der theoretische Grundlagen vermittelt werden sollen, wobei hier für einen Leistungsnachweis sowohl eine Hausarbeit als auch ein Werkstück, mit dem die erworbene Medienkompetenz dokumentiert und die praktische und fachdidaktische Mediennutzung im Unterricht reflektiert werden soll, eingereicht werden müssen, womit die Mediennutzung im Unterricht in den Vordergrund gerückt wird und sich - wie bei dem Projekt Lehr@mt insgesamt - die Frage stellt, ob das Zertifikat eher auf eine Pädagogik mit Medien oder über Medien ausgerichtet ist.

Auf eine Pädagogik der Medien ausgerichtet ist die Studie, die Patrick Bettinger und Franziska Linke von der Universität Hamburg vorgestellt haben. Sie betrachten mit Kerstin Mayrberger Schule als einen Raum, der von soziokulturellen und medienkommunikativen Wandlungsprozessen betroffen ist, und stellen die Frage, wie Schüler/-innen in diesem mediatisierten Handlungsfeld die für ihre aktive Teilhabe an Gesellschaft notwendige Fähigkeit zur Partizipation entwickeln können.

Insgesamt gab es in den zehn Foren der Tagung 29 Beiträge, die über die Lehrerbildung hinaus weitere Aspekte der Schulentwicklung im Kontext von Medienbildung thematisierten; dazu gehörte u.a. das Tablet als Arbeitsgerät in der Schule, die Anwendung von Moodle in der Schule am Beispiel von Lerntagebuch und Podcast, die Schülerreflexion mit Weblogs, die didaktische Qualität von Lern-Apps sowie ein Rahmenkonzept für eine inklusive Medienbildung. Nähere Informationen und Dokumente $\mathrm{zu}$ diesen Beiträgen finden Sie unter: http:// www.uni-kl.de/lbm/dokumentation

\section{Gesine Kulcke}

Akademische Mitarbeiterin in der Abteilung Medienpädagogik. http://www.phludwigsburg.de/14991.html 
Medienpädagogische Grundbildung für pädagogische Fachkräfte

Die Landesgruppe Baden-Württemberg der Gesellschaft für Medienpädagogik und Kommunikationskultur e.V. (GMK) veranstaltete am 30. Oktober 2014 im Evangelischen Medienhaus in Stuttgart einen Fachaustausch zum Thema „Medienpädagogische Grundbildung für pädagogische Fachkräfte“. Die Veranstaltung bezog sich auf die Reihe „Runde Tische Medienbildung“, die vom Staatsministerium Baden-Württemberg 2014 im Rahmen der Initiative „Kindermedienland BadenWürttemberg" durchgeführt wurde. Die Idee $\mathrm{zu}$ einer solchen Reihe geht auf einen Vorschlag zurück, den Prof. Dr. Horst Niesyto auf den 35. Tagen der Stuttgarter Medienpädagogik 2012 machte. Die GMK BadenWürttemberg verband mit der Veranstaltung die Intention, den Diskussionsprozess in den „Runden Tischen Medienbildung“ fachlich zu begleiten und interessierten Kolleginnen und Kollegen eine Gelegenheit zu geben, sich mit ihren Erfahrungen und Ideen aktiv in den Austausch einzubringen.

Das Programm der Veranstaltung beinhaltete u. a. einen Beitrag von Dr. Wolfgang Kreißig (Staatsministerium) zur Entwicklung eines Leitbildes für ein Landesmedienkonzept, von Prof. Dr. Horst Niesyto (PH Ludwigsburg) zur Diskussion über eine Grundbildung Medien im Rahmen der „Runden Tische“ in BW, von Anne Radlinger (Familienbildungsstätte Ulm e.V.) über erfolgreiche Beispiele medienpädagogischer Aus- und Fortbildung von Erzieher/-innen sowie von Dr. Ulrike Bischoff und Dr. Ida Pöttinger (GMK) über Eckpfeiler bzw. Standards medienpädagogischer Grundbildung in der Aus- und Fortbildung.

An der Veranstaltung nahmen insgesamt ca. 35 Personen teil, die aus unterschiedlichen Bereichen kamen: Aktion Jugendschutz BW, Bibliotheken, Evangelische Fachschulen für Sozialpädagogik, Evangelisches Medienhaus GmbH, Fachstelle Medien der Diözese Rottenburg-Stuttgart, Familienbildungsstätten, Gesellschaft für Medienpädagogik und Kommunikationskultur (GMK), Hochschule Esslingen, Jugendamt Stuttgart, Kindermedienland Baden-Württemberg, Kindertagesstätten, Kinomobil e.V., Kita-Fachberatung, Kommunalverband für Jugend und Soziales BadenWürttemberg, Landesanstalt für Kommunikation (LFK), Landesfamilienrat BadenWürttemberg, Landesjugendring BadenWürttemberg, Pädagogische Hochschulen, Staatsministerium Baden-Württemberg, Stadtjugendring Stuttgart.
In zahlreichen Diskussionsbeiträgen wurde wiederholt auf die Notwendigkeit einer nachhaltigen und breitenwirksamen Aus- und Weiterbildung von pädagogischen Fachkräften im Bereich der Medienbildung hingewiesen. Die derzeitigen Förderkonzepte seien in BadenWürttemberg nicht ausreichend. Die anwesenden Vertreter/-innen aus den genannten Einrichtungen konkretisierten die Notwendigkeit einer nachhaltigen medienpädagogischen Grundbildung auf dem Hintergrund ihrer jeweiligen Erfahrungen und akzentuierten unterschiedliche Aspekte.

Im Videoportal der Evang. Landeskirche erschien ein Bericht zur Fachtagung. Der Beitrag von Prof. Dr. Horst Niesyto wird in dieser Ausgabe des Onlinemagazins zugänglich gemacht (Vortragsfolien):

„Grundbildung Medien - Wo stehen wir? Was brauchen wir? Erfahrungen aus den „Runden Tischen“

Die Redaktion 